\title{
Current Status of the Anger Avoidance Model: Recent Empirical Findings and Treatment Considerations
}

\section{Zella E Moore ${ }^{*}$}

Department of Psychology, Manhattan College, Riverdale, New York, USA

*Corresponding author: Zella E Moore, Department of Psychology, Manhattan College, 4513 Manhattan College Parkway, Riverdale, NY 10471, USA, Tel: 718-862-7968; E-mail: zella.moore@manhattan.edu

Rec date: Feb 22, 2016; Acc date: Jun 2, 2016; Pub date: Jun 9, 2016

Copyright: ( 2016 Moore ZE. This is an open-access article distributed under the terms of the Creative Commons Attribution License, which permits unrestricted use, distribution, and reproduction in any medium, provided the original author and source are credited.

\begin{abstract}
The Anger Avoidance Model (AAM), a contemporary theoretical model for understanding the relationship between anger and violence, was presented first in 2008, and since, empirical research has been mostly supportive of its tenets. The AAM essentially purports that individuals prone to violent behaviour typically manifest an aversive developmental history; early maladaptive schemas, which serve as a lens through which one interprets life experience; and poorly developed emotion regulation skills. Such deficits in emotion regulation result in poor tolerance of emotions such as anger, and in turn frequently lead to efforts to avoid or escape the experience of negative emotion, particularly anger, with violent behaviour often the consequence. Based on supported principles within the AAM, contextual anger regulation therapy (CART) was developed specifically to treat clients exhibiting such anger-related violent behaviour. The current article provides a needed update on recent empirical findings that support the AAM, and subsequently discusses the relationship between the AAM and CART, an integrated acceptance-based behavioural intervention that the AAM directly generated.
\end{abstract}

Keywords: Anger; Clinical anger; Anger avoidance model; Violence; Contextual anger regulation therapy

\section{Introduction}

Anger is often been viewed as a maladaptive response to distress. Yet in actuality, the emotion serves an adaptive function, as its basic purpose is to prepare individuals to respond to real threats within the environment [1]. While the experience of anger itself is not a problem, concerns can arise when the experience of anger is excessive or is generalized to situations beyond those in which it is useful. In such circumstances, anger can result in chronic heightened levels of arousal and can be often accompanied by dysfunctional behavioural tendencies. For many people, this heightened duration, intensity, and frequency of anger, which has previously been referred to as clinical anger [2], is associated with violent behaviour, and often culminates in interpersonal, health, occupational, and legal difficulties $[3,4]$.

Considering the substantial negative consequences of clinical anger and its behavioural sequelae, a contemporary theoretical model for understanding the relationship between anger and violent behaviour was developed. The anger avoidance model [2] is founded upon empirical advances regarding the role of deficits in emotion regulation in maladaptive behaviour [5], and the growing use of acceptance-based behavioural approaches in understanding and remediating an ever expanding list of psychopathological conditions [6]. Following the initial publication of this model, a number of important scientific developments have occurred that add to the evidence base for this framework for understanding the anger-violence condition. The current article, therefore, provides an important update on the anger avoidance model based upon recent empirical findings, and subsequently discusses the relationship between the AAM and contextual anger regulation therapy (CART), which is an acceptance- based intervention for clinical anger and violence that the AAM directly spawned.

While there are minor differences between traditional theoretical models that have sought to explain the relationship between anger and violence, by and large they all hold a foundational position that one's personal interpretations of events result in heightened negative affect (anger in particular), and in turn, the experience of this affective state can directly lead to aggressive or violent behaviour [7-11]. This traditional view, which essentially describes a linear and sequential relationship between events, cognition, emotion, and behaviour, has been referred to as a cognitive content specificity model of anger and aggression [12]. As the cognitive content specificity model (in its various iterations) has long been the dominant model for theoretically understanding the relationship between anger and its associated negative consequences, it has likewise informed traditional cognitive behavioural treatments of anger-related violent behaviour. Yet, as has been suggested previously [2], the cognitive content specificity model of anger and aggression has several significant flaws: (a) as anger is a natural human emotion, it seems logically problematic to view the emotion of anger itself as something to be controlled or avoided in order to modify maladaptive anger-related behaviour; (b) cognitivebehavioural treatments for anger and violent behaviour that are based on this theoretical framework have exhibited questionable efficacy, which in turn calls into question the foundational model $[13,14]$; and (c) this theoretical framework has not sufficiently integrated more contemporary findings on emotion and emotional disorders.

\section{The Anger Avoidance Model (AAM)}

Comparatively, the AAM maintains a fundamentally different theoretical position regarding the relationship between anger and violence than does the aforementioned cognitive content specificity model. The AAM suggests that individuals prone to engaging in 
violent behaviour typically present with an aversive developmental history characterized by chronic childhood maltreatment, and a personal temperament style best described as unrestrained and highly reactive [15]. Together, the AAM suggests that these two diatheses in combination result in the development of early maladaptive schemas, which serve as a lens through which one interprets life experience, and poorly developed emotion regulation skills. These deficits in emotion regulation result in poor tolerance of emotions such as anger, and in turn frequently lead to efforts (sometimes extreme) to avoid the experience of negative emotion, particularly anger $[2,16]$. Importantly, the AAM posits that when avoidance of anger is not possible and/or is not effective in reducing or eliminating the experience of anger, violent behaviour often ensues as a way to terminate (i.e., escape from) the perceived intolerable affect.

In recent years, an impressive array of research support has been garnered for AAM hypotheses, which is precisely what warrants the present update. For example, the assertion that there would be a strong relationship between early aversive histories/childhood maltreatment and the perpetration of violence has been consistently noted in the empirical literature [17-19]. Additionally, with regard to another AAM hypothesis, empirical research has consistently supported the relationship between early aversive histories, early maladaptive schemas, anger, and aggressive behaviour [20-22]. Of particular relevance are recent studies by Gay and colleagues [23] and Alfaro and colleagues [24] found that early maladaptive schemas mediated the relationship between childhood emotional abuse and intimate partner violence.

Central to the AAM is the hypothesis that deficits in emotion regulation would be a critical vulnerability for violent behaviour. In fact, recent research has supported the centrality of this vulnerability in a number of ways. First, early aversive histories/childhood maltreatment has been shown to be a direct predictor of deficits in emotion regulation [25-27] as suggested by the AAM. Second, consistent with the AAM hypothesis that deficits in emotion regulation would be central to understanding the relationship between anger and violent behaviour, studies have confirmed the important role of deficits in emotion regulation in the understanding and prediction of violent behaviour [18,19,28-32]. Finally, and of significance, a recent study confirmed that while childhood maltreatment, early maladaptive schemas, deficits in emotion regulation, and the experience of anger together contribute to the manifestation of violence within relationships, consistent with the AAM, deficits in emotion regulation was found to be the essential pathway by which childhood maltreatment results in the manifestation of violence [19]. Consistent with this finding, another recent study found that emotion regulation moderated the relationship between maladaptive schemas and violent behaviour. That is, only in the presence of deficits in emotion regulation did maladaptive schemas demonstrate a relationship with violent behaviour [24]. When seen together, this line of research suggests that following histories of childhood maltreatment, early maladaptive schemas and the experience of anger in the presence of deficits in emotion regulation substantially contribute to later violent behaviour. Importantly, while early maladaptive schemas and the experience of anger are significant contributors to later violent behaviour, moderation and mediation analyses suggest that a substantial portion of their impact on violent behaviour occurs indirectly through deficits in emotion regulation, as hypothesized by the AAM.
It is important to note that deficits in emotion regulation is defined as difficulty with experiencing, tolerating/accepting, and expressing emotion, which in the case of violent behaviour is most typically the emotion of anger. Gardner and Moore [2] have previously suggested that when individuals perceive anger as intolerable, unacceptable, and/or uncomfortable (even when the level of anger is not outside of normative levels), violence may be used as a way to avoid or escape from the experience of the full affective state. This is achieved by either eliminating the aversive stimulus or by changing its form. In fact, studies have suggested that violent behaviour often does function as an escape-based emotion regulation strategy, even among non-clinical populations $[18,30,33]$, a finding consistent with the AAM assertion that escape and/or avoidance strategies are used to minimize or alter the subjective experience of intolerable affective states, and these chronic and maladaptive emotion regulation strategies may at times take the form of violent behaviours $[2,16]$.

It should be noted that there has been one aspect of the AAM that has not yet been sufficiently tested and thus at this point remains purely speculative. Specifically, it is the assertion that an unrestrained and highly reactive temperamental (i.e., biological) vulnerability would interact with the presence of an early aversive history in the development of violent behaviour. A recent study sought to assess this construct by using Gray's Behavioural Inhibition/Behavioural Activation scale [15], but had to remove the measure from analysis due to problems with internal consistency [19]. While other recent studies have utilized the BIS/BAS scales and have found support for the hypothesized relationship between a temperament such as behavioural inhibition and violent behaviour [34,35], future studies will have to carefully choose an appropriate measurement tool for assessing this important construct in concert with emotion regulation and other relevant variables before support for its place in the AAM can be stated. Until then, little can be said beyond the theoretical proposition.

\section{How supported tenets of the AAM can inform treatment}

Given the general support for the AAM model found in recent empirical research, it is reasonable to consider how supported tenets of the AAM can inform the treatment of clients struggling with clinical levels of anger and associated violence. Contextual anger regulation therapy (CART) is a specific intervention model that was developed based on the AAM. The chief goals of CART include the improvement in basic emotion regulation skills of emotional acceptance/tolerance and awareness, and the integration of positive behaviours, which culminates in enhanced quality of life and overall well-being.

The essential strategies utilized in the CART protocol are mindfulness meditation training, cognitive defusion, values-based behavioural activation, emotional acceptance, and interpersonal problem-solving. Each strategy is implemented to help clients function better through enhanced emotion regulation. The goal is not to become less angry (although often, paradoxically, they do), but rather, to function better despite inevitable negative affective states, such as the emotion of anger. This may be best described as a life management intervention rather than an anger management intervention [16]. Indeed, this is the foundation of contextual anger regulation therapy, and is based on key AAM principles.

CART is, at its core, an integrated behavioural treatment approach utilizing components of acceptance-based behavioural therapies [36], mindfulness-based cognitive therapy [37], and a number of strategies most typically associated with emotion-focused therapy [38], and functional analytic psychotherapy [39]. In keeping with the tenets of 
Page 3 of 7

the AAM, CART emphasizes the development of emotion regulation skills. Within CART, emotion regulation can be defined as the awareness, tolerance, utilization, modulation, and appropriate expression of emotion, and CART specifically places an emphasis on the emotion of anger. Of significance, CART's aim is not anger regulation from the traditional standpoint of necessarily reducing or controlling anger. Rather, the use of the term "regulation" is consistent with more contemporary perspectives on emotion. This emotion regulation framework directly informs the CART treatment goals, which can be conceived as:

The capacity to maintain awareness of and tolerate the experience of anger, and come to view emotions in general, and anger in particular, as a normal component of the human experience.

The capacity to understand the motivational aspects of anger, while also being able to modulate anger when necessary (such as when experienced as extreme), in order to more effectively solve problems and manage personal conflict.

Increased behavioural flexibility in response to anger-eliciting situations, which in turn results in improved interpersonal effectiveness and enhanced quality of life.
The CART protocol is comprised of nine distinct modules, which follow a specific sequence. Modules are conceptualized as distinct components, and each requires mastery before moving on to the subsequent module. Each module contains its own targeted treatment objectives, and treatment objectives for each module are built upon the completion of prior module treatment objectives.

The purpose and advantage of this modular approach to psychological intervention is that although the practitioner follows a basic structure of treatment, the protocol still allows for the clinical flexibility required when working in real-world treatment situations.

The nine modules typically require from 10 to 20 sessions to complete, although a briefer structured version of the CART protocol is available, and can be used for contexts in which time for treatment is substantially limited [16].

Below is a brief outline of the nine modules, with each section including strategic goals, strategies, and tactics implemented in each module (Table 1).

\begin{tabular}{|l|l|}
\hline Module & \multicolumn{1}{|c|}{ Theme } \\
\hline 1 & Psychoeducation, Values Identification, and Motivation Enhancement \\
\hline 2 & Using the Therapeutic Relationship to Recognize and Modify Clinically Relevant Behaviour \\
\hline 3 & Developing Mindful Emotion Awareness \\
\hline 4 & Cognitive Defusion and the Reduction of Problematic Rule-governed Behaviour \\
\hline 5 & Understanding Anger and Anger Avoidance \\
\hline 6 & Acceptance and Anger Regulation \\
\hline 7 & Commitment to Values-based Behaviour \\
\hline 8 & Interpersonal Skills Training \\
\hline 9 & Integration, Relapse Prevention, and Treatment Termination \\
\hline
\end{tabular}

Table 1: Cart modules.

\section{Module 1: Psychoeducation, values identification and motivation enhancement}

The principal foci of Module 1 are: (a) how the issues and behaviours that have brought the client into treatment can be explained by the AAM; (b) the benefits and costs of the client's current behavioural patterns; (c) values identification (i.e., what matters most in the client's life); (d) what CART entails, and how it differs from traditional anger management or similar interventions to which the client may have had exposure; and (e) if relevant, how psychological intervention fits into the client's personal directives, such as court mandates.

When the specific objectives of Module 1 are appropriately addressed, the client should experience an enhanced sense of hope, a new way of thinking about current difficulties and future possibilities, and an established, effective working alliance with the therapist [16]. These characteristics have all previously been identified as associated with positive therapeutic outcomes [40]. As may be seen, consistent with being an integrated approach to psychological treatment, there are certainly aspects of early CART intervention that are most often rooted in dialectical behaviour therapy [41]; motivational interviewing [42], acceptance and commitment therapy [36], and more traditional forms of CBT. The central difference is that CART specifically utilizes the anger avoidance model (AAM) as the focal point of these discussions, and integrates aspects of the aforementioned interventions with the clear and empirically informed AAM in order to develop and maintain psychological flexibility and acceptance, and overall wellbeing among clientele.

\section{Module 2: Using the therapeutic relationship to recognize and modify clinically relevant behaviour}

The principal objective of Module 2 is the identification and appropriate therapeutic response to in-session behaviours that are directly connected to identified out-of-session maladaptive behaviours, so that the client can implement new and more functional behaviours. CART incorporates a strong interpersonal component as part of its integrated treatment model. The interpersonal component of CART is 
most closely modeled after Kohlenberg and Tsai's functional analytic psychotherapy [39]. As such, fundamental to CART is the recognition that the client's interpersonal behavioural difficulties will inevitably manifest themselves within treatment sessions, especially sessions that are emotionally charged and frustrating. In particular, the therapist is seen as serving the function of a discriminative stimulus for negative affect, anticipatory cognitions, and maladaptive behaviour.

This interpersonal focus is particularly imperative during the early stages of the treatment process, as in-session client behaviours in response to session scheduling/cancellations, payment, therapist questions/style/gender/race, etc., are often most poignant early in the treatment endeavour. This provides an optimal opportunity and space to note clinically relevant behaviour, which can be defined as withinsession behaviour that corresponds either subtly or directly to the problematic behaviours that occur outside of the therapeutic context.

\section{Module 3: Developing mindful emotion awareness}

There are two principal objectives of Module 3, which are to increase the non-judging, present-moment awareness of emotional experiences, and further, to increase the understanding of one's internal experiences (i.e., cognitions, emotions, and physiological sensations) as personal events that are informative yet transient.

In total, this module seeks to help the client become more comfortable with experiencing, observing, and remaining in longer contact with emotional experience, without reliance on avoidance or escape behaviours. This process starts with psychoeducation about the adaptive value and occasional maladaptive outcomes of emotion, and is followed by psychoeducation about emotion awareness/mindfulness. In this module, the client is then guided through a series of mindfulness-enhancing (meditative) exercises, which: (a) promote disengagement from automatic behaviour in response to one's emotions; (b) promote the ability to non-judgmentally observe and enhance awareness of one's experiences; (c) allow the client to remain in contact with uncomfortable internal experiences despite the urge to flee; and (d) train the client to refocus attention to relevant cues when distracted.

As the development of mindfulness is critical to the CART protocol, a great deal of in- and out-of-session practice is provided and expected. Mindfulness exercises aim to systematically promote the full experience of anger and associated emotions. In addition, greater mindful awareness is intended to disrupt previously learned avoidance/escape behaviours, and therefore directly targets a core process identified in the AAM.

\section{Module 4: Cognitive defusion and the reduction of problematic rule-governed behaviour}

Module 4 maintains three primary objectives, which are to develop an understanding of cognitive fusion and its relationship with aggressive behavioural tendencies; develop an awareness of relevant internal rules (i.e., cognitive schemas) and the automatic behaviours they promote; and develop the capacity to decenter (i.e., distance oneself, without avoidance) from anger-related cognitions and schematic rules.

Cognitive defusion [36] is a critical aspect of Module 4. Cognitive fusion can be defined as the process by which individuals respond to cognitions as though they are absolute truths and realities. When these rules are triggered they are most often associated with a rigid, immediate, and narrow set of behavioural responses. Individuals experiencing difficulties with appropriately responding to frustration and anger most often have very strong, specific, and easily triggered internal rules/schemas. When triggered, these internal rules also trigger automatic behavioural responses, which most often function as a way to avoid or escape from uncomfortable emotions. These behaviours tend to be quite self-destructive.

Conversely, cognitive defusion can be defined as the process by which individuals learn to decenter, or distance themselves from their internal processes. Essentially, the client comes to see their thoughts as a product of what their mind is telling them at any given moment, and not as something that requires an immediate response. The decentering process inherent in cognitive defusion is what some have suggested is the actual mechanism by which cognitive restructuring/ reappraisal strategies (found in traditional forms of CBT) may actually work [37]. It should be noted that cognitive defusion begins with the development of mindfulness and systematically allows the client to slow down, become aware of their internal dialogue, and make informed behavioural choices that are not automatic and based upon previously over-learned rules. It is also important to note cognitive defusion does not utilize logical analysis or Socratic dialogue with the intent of changing, reducing, or eliminating specific thoughts or rules, as is typical in traditional cognitive-behavioural treatments. Instead, the client develops an enhanced capacity to notice and let go of internal dialogue, and see internal experience as something that comes and goes in one's mind, something that can be observed and tolerated, and thus something that does not necessitate immediate action.

\section{Module 5: Understanding anger and anger avoidance}

Module 5 maintains several key goals, which are to: (a) to develop a different understanding of the emotion of anger as a normal and unavoidable emotion, (b) to understand common misconceptions of anger (e.g., it inevitably results in violence if not controlled; venting is healthy), (c) to understand the personal costs of efforts to avoid or escape from the experience of anger; and (d) to develop an enhanced capacity to tolerate being angry without engaging in avoidance or escape behaviours.

Ultimately, the goal is to develop a greater comfort with feeling angry, instead of believing that one needs to feel less anger. As one's personal experience of anger is normalized and better tolerated, the client increasingly recognizes that the real problem has been the efforts they have engaged in to not feel anger, and not the actual anger itself. This initiates the critical process by which anger avoidance/escape behaviours are naturally and systematically exchanged for the opposite-action [41] of experience and non-response... and this learning process results in significant resultant positive outcomes.

\section{Module 6: Acceptance and anger regulation}

Focusing on acceptance and anger regulation, Module 6 helps to further develop the willingness to tolerate/accept the experience of anger, even when intense; and seeks to clearly differentiate behaviours directed at avoiding the experience of anger from those that are in the service of pursuing personal values (i.e., behaviour that promotes a life consistent with one's own goals and values). Further, during Module 6, clients learn to modulate (i.e., down-regulate) anger when necessary, not simply to feel better, but instead, with the intent of actively pursuing personal values. In essence, herein, the client increasingly engages in systematic in- and out-of-session exposure-based exercises, 
in which anger-evoking situations of varying degrees of difficulty are fully experienced and tolerated.

There are those times, however, in which intense anger can interfere with optimal behavioural responding, and therefore, there may be the need to develop appropriate self-soothing abilities. The distinction between appropriate self-soothing and avoidance-based self-soothing can be seen in the primary function of each. For instance, a client's use of a brief relaxation exercise to reduce his anger, with the direct intent of continuing on with a difficult but important conversation with his employer following the relaxation exercise, is certainly different in its function than the same client using the relaxation technique to reduce anger and prematurely terminate (and thus escape from) that difficult dialogue. While strategies intended to help reduce the intensity of anger can be useful, it is important that the use of any self-soothing techniques be carefully monitored, as not to inadvertently promote a subtle form of avoidance. As such, it is important that these skills only be introduced following the development of anger distress tolerance.

\section{Module 7: Commitment to values-based behaviour}

Module 7's focus on commitment to values-based behaviour adds a vital component to the treatment protocol. This module seeks to help clients: (a) recognize the connection between identified personal values and specific behaviours; (b) recognize that intense anger can actually occur at the same time as effective values-based behaviours; (c) develop a targeted behavioural activation plan; and (d) identify additional skill deficits that may be in need of further development or refinement.

As anger tolerance develops over time, the therapeutic focus shifts to the development of an ongoing commitment to pursuing meaningful personal values through the activation and maintenance of values-based behaviours. Since goals and values are not the same, asserting the distinction between goals and values is especially important at this juncture. Specifically, goals are defined as having an identifiable end point, while values have no real end point, and therefore require enduring attention and action. Herein, the therapist encourages a willingness to engage in actions that are in the service of stated values (even while at times being angry), and as such, the client is encouraged to increasingly confront important yet still avoided life situations. By implementing a systematic behavioural action plan, new emotional meanings can be developed, especially when important situations are dealt with directly instead of being avoided. In turn, a greater sense of personal effectiveness is established. Of significance, as important life situations are now confronted and personal effectiveness arises in some key areas, skill deficits such as those pertaining to communication, conflict management, and problem-solving may then be identified as requiring some attention. This is thus an interesting time of therapeutic exploration and leads to the consideration of key components to integrate into Module 8.

\section{Module 8: Interpersonal skills training}

Accordingly, Module 8 seeks to develop interpersonal problemsolving skills, communication and assertiveness skills, and conflict management skills, as needed and appropriate for the particular client. Such skills are taught and practiced within sessions, and betweensession work on these skills will be integrated for the development of long-lasting results. Both the in-session and between-session skillsbased work is met with valuable therapist feedback, thus allowing for appropriate behavioural shaping. These skills are the focus of treatment at this particular point in the CART protocol because they can most effectively be integrated following the development of mindful emotional awareness and anger tolerance. At this time, the development of these more effective and "positive" skills/behaviours further increases the likelihood that the client will welcome naturally occurring positive reinforcement within his or her community, and will in turn be more likely to maintain the use of values-based behavioural choices in the future.

\section{Module 9: Integration, relapse prevention and treatment termination}

As the final module in the CART program, the objectives of Module 9 necessarily focus on skill integration, relapse prevention, and issues associated with the termination of treatment. The specific foci of the module are: (a) the identification of potential future anger-inducing triggers, and in vivo practice in responding to such triggers; (b) the consideration of effective responses to possible challenges/lapses; (c) the formation of an action plan for continued future use of CART principles following completion of treatment; and (d) treatment termination.

The essential purpose of this module is the formulation of a clear plan to tackle the stressors that one can expect to face, how to respond to inevitable lapses, and to systematically discuss the termination of treatment as a beginning and not an ending, somewhat akin to a graduation. The development of an action plan for the continued use of CART principles and strategies is key, and the action plan should include continued self-monitoring, self-reflection, and self-correction. With the therapist's guidance, the client will work to identify and practice effective responses to likely anger-inducing situations that have not received attention during treatment; discuss the likelihood of, and ways of responding to inevitable lapses; and in turn, develop specific plans to keep these normal and inevitable lapses from devolving into relapses involving old behavioural patterns.

Lastly, since clients with aggression-related difficulties most often have had a history of childhood abandonment, neglect, or abuse (as described and tested in the AAM), it is important for the therapist to provide a supportive dialogue about the conclusion of the formal therapy process, and support and reinforce clients' readiness to utilize CART principles on their own. This treatment protocol assumes that treatment will be relatively short-term, and as such, termination of treatment should be openly and honestly addressed in order to promote a corrective and healthy interpersonal experience.

While still early in its development, recent preliminary studies have suggested the utility of the CART treatment model with adults court mandated for violent behaviour and school-based programs focusing on youth demonstrating difficulties with anger and aggressive behaviour [43-45]. Future studies will need to engage in large traditional randomized controlled trials before clear statements of efficacy can be made; however, considering the troubling lack of efficacy demonstrated by more traditional interventions, these early studies are certainly promising.

\section{Conclusion}

This article sought to present the anger avoidance model in the context of recent studies that have continued to offer broad support to its fundamental tenets. In fact, research continues to offer support to the understanding of the way childhood maltreatment, maladaptive schemas, anger, and deficits in emotion regulation interact in the 
development of violent behaviour. In particular, the essential pathway between childhood maltreatment and violent behaviour has increasingly been demonstrated to be emotion dysregulation.

In turn, as the propositions of the anger avoidance model have gained increasing empirical support, a psychological treatment for anger-related violent behaviour has been developed based on the tenets of the AAM. Known as contextual anger regulation therapy (CART), the 9-module protocol demonstrates exciting possibilities in the treatment of this challenging clinical condition. Described in detail herein, it is hoped that this article will lead both clinicians and researchers to consider this innovative behavioural approach to angerrelated violent behaviour.

\section{References}

1. Kemper TD (1987) How many emotions are there? Wedding the social and the autonomic components. Amer J Socio 93: 263-289.

2. Gardner FL, Moore ZE (2008) Understanding clinical anger and violence: the anger avoidance model. Behav Modif 32: 897-912.

3. Vecchio DT, O'Leary D (2004) Effectiveness of anger treatments for specific anger problems: A meta-analytic review. Clin Psychol Rev 24: 15-34.

4. Kassinove H, Sukhodolsky DG (1995) Anger disorders: basic science and practice issues. In Kassinove $\mathrm{H}$ (ed.), Anger disorders: Definition, diagnosis, and treatment. Taylor \& Francis, Washington DC, USA, pp. 1-26.

5. Aldao A, Nolen-Hoeksema S, Schweizer, S (2010) Emotion regulation strategies across psychopathology: A meta-analytic review. Clin Psychol Rev 30: 217-237.

6. Kashdan TB, Barrios V, Forsyth JP, Steger MF (2006) Experiential avoidance as a generalized psychological vulnerability: Comparisons with coping and emotion regulation strategies. Behav Res Ther 44: 1301-1320.

7. Baumeister RF, Smart L, Boden JM (1996) Relation of threatened egotism to violence and aggression: the dark side of high self-esteem. Psychol Rev 103: 5-33.

8. Beck AT (1999) Prisoners of hate: The cognitive basis of anger, hostility and violence. Harper Collins, New York, USA.

9. Berkowitz L (1989) Frustration-aggression hypothesis: examination and reformulation. Psychol Bull 106: 59-73.

10. Berkowitz L, Harmon-Jones E (2004) Toward an understanding of the determinants of anger. Emotion 4: 107-130.

11. Novaco RW (1975) Anger control. Lexington, MA: Lexington, USA.

12. Smith PN, Mumma GH (2008) A multi-wave web-based evaluation of cognitive content specificity for depression, anxiety and anger. Cogn Ther Res 32: 50-65.

13. Santanello AP, Gardner FL, Moore ZE (2008) Reconsidering the efficacy of psychological treatments for clinical anger: A criterion-based, qualitative review. Unpublished manuscript.

14. Olatunji BO, Lohr JM (2005) Nonspecific factors and the efficacy of psychosocial treatments for anger. The Scien Rev Ment Hea Pract 3.

15. Gray JA (1994) Three fundamental emotion systems. In Ekman P, Davidson RJ, The nature of emotion: Fundamental questions, Oxford University Press, New York, USA.

16. Gardner FL, Moore ZE (2014) Contextual anger regulation therapy: A mindfulness and acceptance-based approach. Routledge/Taylor \& Francis, New York, USA.

17. Bevan E, Higgins DJ (2002) Is domestic violence learned? The contribution of five forms of child maltreatment to men's violence and adjustment. J Fam Viol 17: 223-245.

18. Gratz KL, Paulson A, Jakupcak M, Tull MT (2009) Exploring the relationship between childhood maltreatment and intimate partner abuse: Gender differences in the mediating role of emotion dysregulation. Violence Vict 24: 68-82.
19. Smyth E, Gardner FL, Marks D, Moore ZE (2016) An exploration of the mediators between childhood maltreatment and intimate partner violence. Violence Vict.

20. Cohen DJ., Eckhardt CI, Schagat KD (1998). Attention allocation and habituation to anger-related stimuli during a visual search task. Aggr Behav 24: 399-409.

21. Smyth EJ, Dettore M, Gardner FL, Moore ZE (2010) Emotion dysregulation as a mediator between early maladaptive schemas and anger. Annual convention of the Association for Behavioural and Cognitive Therapies, San Francisco, CA, USA.

22. Wenzel A, Lystad C (2005) Interpretation biases in angry and anxious individuals. Behav Res Ther 43: 1045-1054.

23. Gay LE, Harding HG, Jackson JL, Burns EE, Baker BD (2013) Attachment style and early maladaptive schemas as mediators of the relationship between childhood emotional abuse and intimate partner violence. J Aggress Maltreat Trauma 22: 408-424.

24. Alfaro W, Bueno C, Marks DR, Gardner FL, Moore, et al. (2015) Cognitive-affective schemas mediate the relationship between childhood trauma and intimate partner psychological and physical aggression among adult females. Annual convention of the Association for Behavioural and Cognitive Therapies, Chicago, IL, USA.

25. Gardner FL, Moore ZE, Dettore MT (2014) The relationship between anger, childhood maltreatment, and emotion regulation difficulties in intimate partner and non-intimate partner violent offenders. Behav Modif 38: 779-800.

26. Castro BOD, Merk W, Koops W, Veerman JW, Bosch JD (2005) Emotions in social information processing and their relations with reactive and proactive aggression in referred aggressive boys. J Clin Child Adolesc Psychol 34: 105-116.

27. Sullivan TP, Meese KJ, Swan SC, Mazure CM, Snow DL (2005) Precursors and correlates of women's violence: Child abuse traumatization, victimization of women, avoidance coping, and psychological symptoms. Psych Wom Quar 29: 290-301.

28. Bell KM, Naugle AE (2008) Intimate partner violence theoretical considerations: Moving toward a contextual framework. Clin Psychol Rev 28: 1096-1107.

29. Bliton CF, Wolford-Clevenger C, Zapor H, Elmquist J, Brem MJ, et al. (2015) Emotion dysregulation, gender, and intimate partner violence perpetration: An exploratory study in college students. J Fam Viol 31: 371-377.

30. Jakupcak M, Lisak D, Roemer L (2002) The role of masculine ideology and masculine gender role stress in men's perpetration of relationship violence. Psych Men \& Mascul 3: 97-106.

31. Shorey RC, Brasfield H, Febres J, Stuart GL (2011) An examination of the association between difficulties with emotion regulation and dating violence perpetration. J Aggress Maltreat Trauma 20: 870-885.

32. Shorey RC, Brasfield H, Febres J, Stuart GL (2011) An examination of the association between difficulties with emotion regulation and dating violence perpetration. J Aggress Maltreat Trauma 20: 870-885.

33. Liu E, Roloff ME (2015) To avoid or not to avoid: When emotions overflow. Comm Res Rep 32: 332-339.

34. Rohlfs P, Ramírez JM (2006) Aggression and brain asymmetries: A theoretical review. Aggr Viol Behav 11: 283-297.

35. Sotelo JM, Babcock JC (2013) BIS/BAS variables as moderators of the rumination-intimate partner violence link. J Fam Viol 28: 233-242.

36. Hayes SC, Strosahl KD, Wilson KG (2012) Acceptance and commitment therapy: The process and practice of mindful change. Guilford Press, New York, USA.

37. Segal ZV, Williams JMG, Teasdale JD (2002) Mindfulness based cognitive therapy for depression: A new approach to preventing relapse. Guilford Press, New York, USA.

38. Elliot R, Watson JC, Goldman RN, Greenberg LS (2004) Learning emotion focuses therapy: The process experiential approach to change. American Psychological Association, Washington DC, USA. 
Citation: Moore ZE (2016) Current Status of the Anger Avoidance Model: Recent Empirical Findings and Treatment Considerations. J Foren Psy 1: 107. doi:10.4172/2475-319X.1000107

Page 7 of 7

39. Kohlenberg RJ, Tsai M (1993) Functional analytic psychotherapy: A radical behavioural approach to treatment and integration. J Psych Integr 4: 175-201.

40. Castonguay LG, Beutler LE (2006) Principles of therapeutic change: a task force on participants, relationships, and techniques factors. J Clin Psychol 62: 631-638.

41. Linehan MM (1993) Skills training manual for treating borderline personality disorder. Guilford Press, New York, USA.

42. Miller WR, Rollnick S (2002) Motivational interviewing: Preparing people for change (2nd edn.), Guilford Press, New York, USA.

43. Thom A, Almeida A, Gardner FL (2014) Contextual anger regulation therapy for youth: A pilot study. Annual convention of the National Association of School Psychologists, Washington DC, USA
44. Moore ZE, Gardner FL, Storey MA, Almeida A (2013) Probation officer satisfaction with a contemporary approach to treatment of interpersonal violence: Contextual anger regulation therapy. Annual convention of the Association for Behavioural and Cognitive Therapies, Nashville, TN, USA.

45. Gardner FL, Moore ZE, Pess R (2012) A pilot study examining the effectiveness of anger regulation therapy (ART) for the treatment of interpersonal partner violence. Annual convention of the Association for Behavioural and Cognitive Therapies, National Harbor, Maryland, USA. 